\title{
Estudo de base populacional sobre as condições periodontais e determinantes socioeconômicos em adultos residentes no município de Guarulhos (SP), Brasil, 2006
}

\section{Population based study on periodontal conditions and socioeconomic determinants in adults in the city of Guarulhos (SP), Brazil, 2006}

Trabalho realizado no município de Guarulhos (SP) e Faculdade de Odontologia da Universidade de São Paulo (FO-USP) - São Paulo (SP), Brasil.

Fonte de financiamento: nenhuma.

Correspondência: Antônio Carlos Frias - Av. Prof. Lineu Prestes, 2227 - Cidade Universitária CEP: 05508-900 - São Paulo (SP), Brasil - E-mail: acfrias@usp.br

Conflito de interesse: nada a declarar.

\section{Resumo}

Objetivo: Este estudo descreveu a prevalência de condições periodontais e sua associação com variáveis demográficas, socioeconômicas e comportamentais em adultos de 35 a 44 anos residentes no município de Guarulhos, São Paulo, 2006. Métodos: Foi realizado estudo transversal de base populacional com delineamento amostral por conglomerados. Os inquéritos e os exames foram realizados nos domicílios sorteados. Para avaliação da condição periodontal foi utilizado o índice Community Periodontal Index (CPI). Os dados foram apurados pelo programas EPIINFO 6 e STATA 10. Para o modelo de associação empregou-se a razão de prevalências (RP), calculada por meio de análise de regressão de Poisson, com correção para delineamento de amostras complexas e ponderação pelo peso populacional. Resultados: Foram examinadas 263 pessoas em 237 domicílios. No estudo multivariado, com modelo ajustado para amostras complexas e ponderação populacional, a prevalência de sangramento gengival e cálculo dentário esteve associada significativamente ao fato de ser homem $(\mathrm{RP}=1,12$, $\mathrm{p}=0,04)$, fumante $(\mathrm{RP}=1,11, \mathrm{p}=0,01)$, ter menos de oito anos de estudo $(\mathrm{RP}=1,14$, $\mathrm{p}=0,01)$ e não visitar o cirurgião-dentista (CD) há mais de dois anos $(\mathrm{RP}=1,19$, $\mathrm{p}=0,00$ ). Para a presença de bolsa periodontal, houve associação com adultos que relataram o hábito de fumar $(\mathrm{RP}=1,71, \mathrm{p}=0,02)$ e apresentavam mais de dois anos sem frequentar o $C D(R P=1,97, p=0,03)$. Conclusão: Houve maior prevalência $(62,7 \%)$ de indivíduos com sangramento gengival e cálculo dentário em relação à presença de bolsas periodontais rasas ou profundas $(26,6 \%)$; as primeiras, reversíveis, têm possibilidades de resolução por meio de procedimentos de atenção básica em saúde bucal.

Palavras-chave: índice periodontal; estudos transversais; amostragem por conglomerados; fatores socioeconômicos; epidemiologia; periodontia. 


\section{Abstract}

Objective: This study described the prevalence of periodontal conditions and assessed its association with social, economical and behavioral variables in adults aged 35 to 44 years old, residents in the city of Guarulhos, State of Sao Paulo, 2006. Methods: This study was a population based survey with cluster sampling. Data collecting and exams were done at houses drew at random. The Community Periodontal Index was used to assess the periodontal condition. Data analysis used EPIINFO 6 and STATA 10 softwares. The assessment of association used the prevalence ratio (PR), as calculated by Poisson regression models with complex sampling and weighted sample adjustments. Results: The total number of people was 263 in 237 domiciles. In the multivariate model, the prevalence of gingival bleeding and dental calculus was significantly associated to being male ( $\mathrm{PR}=1.12, \mathrm{p}=0.04)$, smoking $(\mathrm{PR}=1.11$, $\mathrm{p}=0.01)$, school level of less than eight years of study (PR=1.14, $\mathrm{p}=0.01$ ), and who have not received dental care for more than two years $(\mathrm{PR}=1.19, \mathrm{p}<0.01)$. As for the presence of periodontal pocket, associated factors were smoking $(\mathrm{PR}=1.71, \mathrm{p}=0.02)$ and who have not received dental care for more than 2 years $(\mathrm{PR}=1.97, \mathrm{p}=0.03)$. Conclusion: We observed a higher prevalence $(62.7 \%)$ of individuals with gingival bleeding and dental calculus in relation to the presence of shallow or deep periodontal pockets $(26,6 \%)$. As shallow pockets are reversible, their treatment may be resolute in programs of primary dental care.

Keywords: periodontal index; cross-sectional studies; cluster sampling; socieconomic factors; epidemiology; periodontics.

\section{Introdução}

Levantamentos epidemiológicos em saúde bucal são importantes instrumentos para determinar com assertividade sobre a alocação de recursos e propor medidas preventivas aos agravos, principalmente os de caráter reversíveis, que são detectados precocemente, o que propicia a prática de ações assistenciais primárias. $\mathrm{O}$ diagnóstico dos problemas que afetam a comunidade, com a identificação dos grupos mais vulneráveis e expostos aos riscos de adoecer, torna-se uma importante ferramenta para o planejamento das estratégias de políticas públicas e planos de ação em promoção de saúde bucal.

Populações de baixa renda econômica e com pouca escolaridade são mais expostas a desenvolver agravos periodontais ${ }^{1,2}$, devido ao baixo acesso aos bens de higiene pessoal e bucal e acesso esporádico aos serviços de saúde, tanto público como privado.

Condições periodontais, em particular as periodontites severas são uns dos responsáveis pelas perdas dentárias em adultos e idosos ${ }^{3}$.

Um dos mais comuns problemas periodontais apresentados pela população é a gengivite, que é uma resposta inflamatória do organismo frente à agressão da placa bacteriana, e o primeiro sinal detectável é o sangramento gengival. Muitos indivíduos, frente aos primeiros sinais de sangramento durante a escovação, suspendem os hábitos de higiene bucal por acreditarem que a escovação estaria provocando o sangramento, perpetuando um problema reversível.

Em 2003, um levantamento nacional realizado pelo Ministério da Saúde ${ }^{4}$ sobre as "Condições de Saúde Bucal da População Brasileira 2002-03", examinou as condições dentárias de 13.431 adultos de 35 a 44 anos de idade empregando o índice Community Periodontal Index (CPI), segundo metodologia recomendada pela Organização Mundial da Saúde(OMS). Na região sudeste $(\mathrm{n}=2.340)$, apenas $23,0 \%$ dos examinados apresentavam todos os sextantes hígidos; 
9,7\% apresentavam sangramento identificado pela sondagem; $42,8 \%$ apresentavam cálculo dentário; 10,1\% apresentam bolsa periodontal (3,5 a $5,5 \mathrm{~mm})$; e $2,4 \%$ apresentam bolsa profunda (mais de $5,5 \mathrm{~mm}$ ). A porcentagem de sextantes excluídos foi de $12,0 \%$, resultado da mutilação dentária causada pela associação da cárie e da doença periodontal.

O município de Guarulhos é a cidade mais populosa e com a primeira economia do estado, excluindo a capital São Paulo. Está localizado na região metropolitana da grande São Paulo, em uma área de $334 \mathrm{Km}^{2}$, com uma população de 1.258.205 habitantes em 2006, segundo dados da Fundação Seade $^{5}$. São 190.016 adultos de 35 a 44 anos de idade residentes em Guarulhos, ou seja, $15,1 \%$ da população total do município, sendo que desses $51,2 \%$ são mulheres.

$\mathrm{O}$ índice de desenvolvimento humano municipal é considerado alto (IDH$\mathrm{M}=0,798$ ), a taxa de analfabetismo na população acima de 15 anos é de 6,3\%, e 94,7\% e $77,1 \%$ dos domicílios têm acesso à água tratada e esgoto, respectivamente, mas esse último não recebe qualquer tratamento ${ }^{5}$.

Em relação aos serviços públicos de saúde, o município possui 64 unidades básicas de saúde (UBS), das quais 29 trabalham com estratégia de saúde da família. Em 2006, 127 cirurgiões-dentistas atuavam em UBS e em quatro Centros de Especialidades Odontológicas, que prestam assistência complementar à atenção básica.

A saúde bucal de adultos vem sendo explorada na literatura a partir dos dados do último levantamento epidemiológico em saúde bucal ${ }^{4}$, realizado entre os anos de 2002 e $2003^{6-8}$. Até então, essa população era estudada a partir da coleta de dados entre servidores ou usuários de serviços públicos ${ }^{6,9}$ ou de determinadas categorias profissionais ${ }^{10}$.

Devido ao fato de a doença periodontal ter uma alta prevalência, principalmente entre a população adulta ${ }^{4}$, e levando-se em consideração que esse agravo é um dos fatores que levam as pessoas ao edentulismo $^{3,6,11}$, conhecer sua prevalência em parâmetros populacionais e identificar possíveis fatores relacionados amplia a possibilidade de atuação no âmbito da saúde coletiva, o que pode contribuir para a diminuição da mutilação dentária resultante da doença periodontal.

O objetivo deste estudo é descrever a prevalência das condições periodontais: sangramento gengival, cálculo dentário e bolsa periodontal e avaliar sua associação com variáveis socioeconômicas no grupo etário de 35 a 44 anos de idade residentes no município de Guarulhos, 2006.

\section{Materiais e métodos}

Em outubro de 2006, realizou-se um levantamento epidemiológico das condições de saúde bucal no município de Guarulhos, para os seguintes agravos, cárie, fluorose, periodontopatias, uso e necessidade de prótese dentária, em diferentes grupos etários (adolescentes, adultos e idosos).

$\mathrm{O}$ presente estudo concentrou-se em descrever e analisar os problemas periodontais no grupo etário dos adultos (35 a 44 anos) residentes no município de Guarulhos. Esta foi a primeira pesquisa populacional realizada nesse grupo etário no município. $\mathrm{O}$ estudo foi do tipo transversal com delineamento amostral por conglomerado, sendo os exames realizados nos domicílios sorteados.

\section{Delineamento da amostra}

Para o cálculo do tamanho da amostra recorreu-se à equação proposta por Lwanga e Lemeshow ${ }^{12}$ para prevalência do agravo em populações infinitas e agregou-se na fórmula o efeito do desenho, ajustando assim o tamanho amostral ao modelo por conglomerado em dois estágios de sorteio. Também foi incluída na fórmula a taxa de não-resposta (TNR) ${ }^{13}$.

Para obtenção do cálculo amostral são necessários alguns parâmetros da doença e foram considerados os dados obtidos no Levantamento Epidemiológico de base nacional para a região Sudeste, onde a prevalência de doença periodontal 
em adultos de 35 a 44 anos foi de $77,0 \% 4$. Estabeleceu-se também um nível de significância de $5 \%(\alpha=0,05)$, uma margem de erro aceitável $7,5 \%(\varepsilon=0,075)$, um efeito de desenho de 2,0 e uma taxa de não resposta (percentual estimado de perda de elementos amostrais) de 20,0\% (TR=0,80). Assim sendo, o tamanho da amostra esperada foi de 302 indivíduos.

O delineamento do sorteio adotado no estudo foi o de conglomerado em dois estágios. Na primeira etapa foram sorteados sistematicamente 60 setores censitários (Figura 1 - Unidade Primária de Amostragem - UPA) ${ }^{14}$ no universo de 1.339 setores que compõem o município de Guarulhos, onde estão distribuídos 299.979 domicílios. No segundo estágio, foram sorteados domicílios de cada um dos setores sorteados, sendo o domicílio a Unidade Secundária de Amostragem (USA).

Cada setor censitário teve igual probabilidade de ser sorteado, sendo a fração de sorteio no primeiro estágio de 0,0448 (fl=total de setores sorteados dividido pelo total de setores do município: $\mathrm{fl}=60 / 1.339=0,0448)$.

Após identificação dos setores censitários sorteados, ocorreu o arrolamento desses e a identificação dos domicílios em cada um dos setores. Para a definição do número de domicílios sorteados para a obtenção da amostra de adultos, estimou-se em quatro adultos no grupo etário para cada dez imóveis ocupados (sendo "d" o número de domicílios elegíveis onde seria possível encontrar a amostra desejada, $d=302 *(10 / 4)=755)$.

Dividiu-se o número de domicílios por setor censitário $(755 / 60=12,58)$, valor arredondado para 13. Seria necessário, então, o sorteio de 13 domicílios em cada setor, ou seja, $13 * 60=780$ (a fração de sorteio no segundo estágio foi f2 $=13 /$ total de domicílios no setor sorteado). Foram sorteados pelo método sistemático 13 domicílios em cada setor que, após identificação nos mapas censitários, eram visitados pelos examinadores e anotadores. Caso fossem identificados indivíduos na faixa

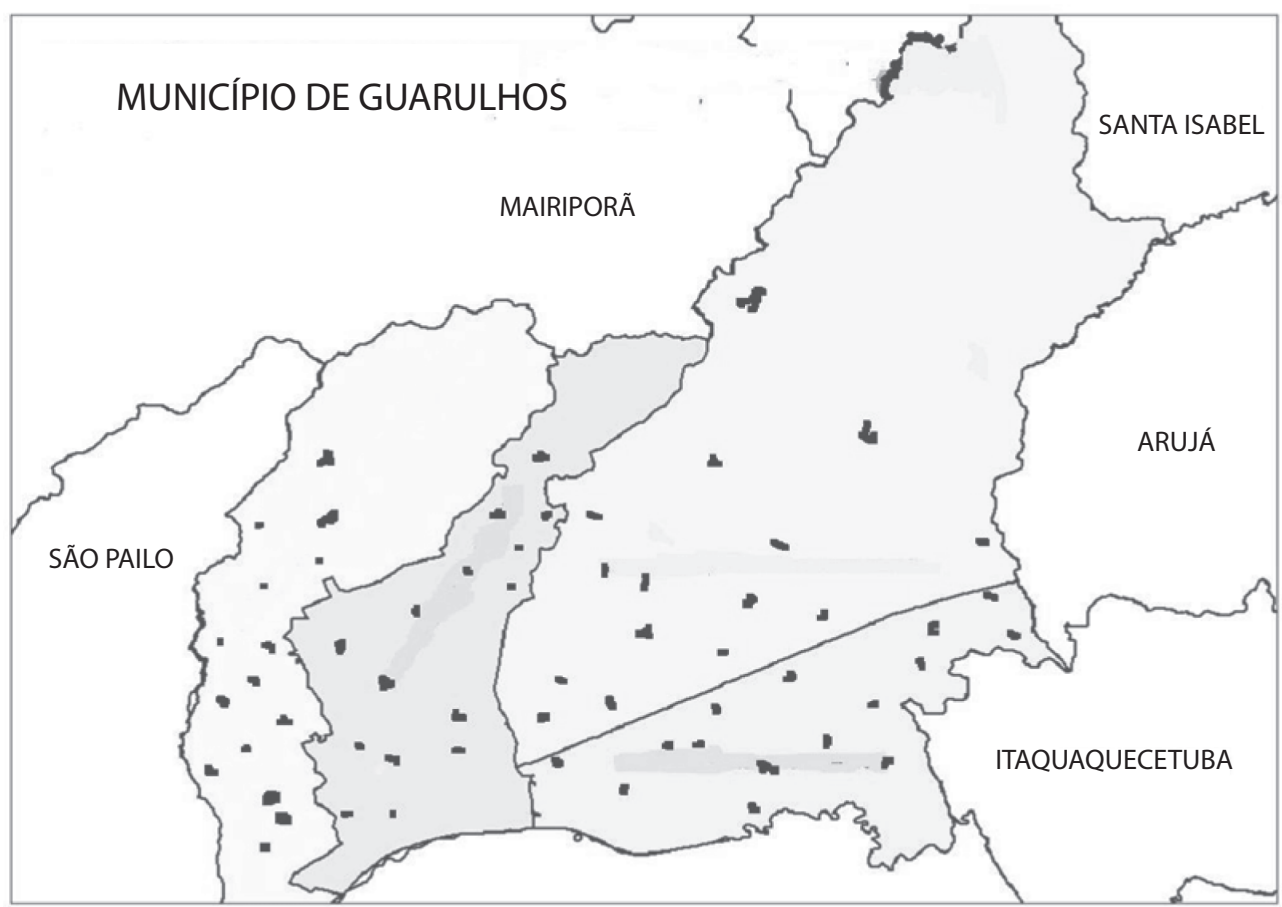

Figura 1. Setores censitários sorteados para levantamento epidemiológico de saúde bucal no município de Guarulhos (SP), 2006

Figure 1. Census tracts selected to the oral health epidemiological survey in the city of Guarulhos (SP), 2006 
etária de interesse nos domicílios visitados, todos eram convidados a participar da pesquisa. Caso concordassem, após a assinatura do Termo de Consentimento Livre e Esclarecido, eram entrevistados e examinados. Quando esses não estavam presentes ou não estavam disponíveis para a realização da pesquisa, a equipe realizava o agendamento prévio em horário favorável ao indivíduo e retornava à residência mais duas vezes; caso a entrevista não fosse realizada o indivíduo era incluído no cálculo da Taxa de Não Resposta.

A amostra foi realizada em dois estágios, por conglomerado, com a atualização do tamanho do setor censitário (número de domicílios) na etapa de arrolamento do setor em campo, e com sorteio igual de domicílios em cada setor. A fração de amostragem geral é a multiplicação da fração de amostragem de sorteio no primeiro e segundo estágio $\left[\mathrm{f}=\mathrm{f} 1^{*} \mathrm{f} 2\right]$, sendo que o peso de ponderação do plano de amostragem foi considerado o inverso da fração de amostragem $(\mathrm{w}=1 / \mathrm{f})^{14}$. Além desse recurso, levou-se em consideração o efeito do conglomerado no processo de análise estatística dos resultados.

\section{Calibração dos examinadores}

Formaram-se 12 equipes de examinadores (cirurgiões-dentistas) e anotadores (auxiliares de saúde bucal). A calibração foi estruturada em duas etapas, uma teórica (8 horas) e uma prática (32 horas), em um Centro de Especialidade Odontológica do município. Para o controle de reprodutibilidade das observações efetuadas e avaliação da concordância inter e intraexaminadores dos agravos periodontais empregou-se o coeficiente kappa; ao todo foram examinados e reexaminados 46 indivíduos.

\section{Definição das variáveis}

Para a avaliação da condição periodontal foi utilizado o índice CPI, segundo os critérios de diagnóstico estabelecidos pela $\mathrm{OMS}^{15}$. Cada sextante da boca, examinados pelos dentes índices 17, 16, 11, 26, 27, 47, $46,31,36$, e $37^{15}$, recebe uma classificação de acordo com a condição periodontal dos mesmos. O sextante pode ser saudável (CPI=0); ou apresentar sangramento gengival à sondagem $(\mathrm{CPI}=1)$; cálculo dentário (CPI=2); bolsa periodontal de 3,5 a $5,5 \mathrm{~mm}$ (CPI=3); bolsa periodontal profunda com mais de 5,5 mm (CPI=4) ou ser excluído, por apresentar menos de dois dentes em condições de exame, ou todos os dentes extraídos (CPI=X). Foi fator de exclusão para esta pesquisa os indivíduos desdentados totais.

Além da ficha de exame bucal das condições periodontais foi utilizado um questionário respondido pelo indivíduo para compor as variáveis independentes como: sexo, idade, cor da pele e hábito de fumar, tempo que frequentou a escola (anos de estudo), renda familiar e pessoal em reais (posteriormente transformada em salários mínimos - SM), informações referentes à residência para construir a variável de aglomeração domiciliar (número de pessoas por cômodo) e questões sobre acesso aos serviços de saúde, tempo da última visita, tipo de serviço procurado e se apresentou dor de dente nos últimos seis meses anteriores à pesquisa.

A coleta de dados foi do tipo domiciliar e o local de exame na residência foi o cômodo com melhor iluminação natural. O indivíduo sentava-se em uma cadeira e o examinador, em pé, posicionava-se à frente dele; ao lado o anotador registrava os dados na ficha. O examinador usava os equipamentos de proteção individual e, para o exame bucal, utilizou-se sonda do tipo CPI e espelho plano $n^{\circ} 5$, previamente esterilizados.

O presente estudo teve aprovação do Comitê de Ética em Pesquisa da Faculdade de Odontologia da Universidade de São Paulo (USP) - Parecer de Aprovação 86/06.

\section{Análise estatística}

Para a digitação e a apuração dos dados, utilizou-se o programa EPIINFO 6 versão 6.04, incluindo o modulo CSAMPLE para análise bivariada dos dados provenientes 
de delineamentos complexos de amostragem. A associação multivariada dos dados foi executada pelo programa STATA 10 utilizando-se a Regressão de Poisson, com ajuste para amostras por conglomerados; utilizou-se a linearização de Taylor com ajuste para UPA e com peso de ponderação populacional (weight) para os dois estágios de sorteio da amostra ${ }^{16}$.

Analisaram-se duas variáveis de desfecho: a prevalência da condição periodontal (presença de sangramento gengival - CPI=1 e cálculo dentário - CPI=2); e a prevalência de bolsa periodontal (considerada rasa de 3,5 a $5,5 \mathrm{~mm}-\mathrm{CPI}=3$ ou profunda quando maior que 5,5 mm - CPI=4). Consideraram-se os dois desfechos tendo em vista que a presença de sangramento e cálculo são situações reversíveis, ao passo que a presença de bolsa periodontal representa a consolidação e severidade da doença, sendo irreversível.

Empregou-se a Razão de Prevalência (RP) e o respectivo intervalo de confiança de $95 \%$.

As variáveis independentes foram dicotomizadas para a realização da associação bivariada e multivariada. São elas: grupo etário; gênero; cor da pele; hábito de fumar; anos de estudo; renda familiar em salários mínimos [SM=R \$ 350,00]; aglomeração familiar; tempo desde a última visita ao dentista; tipo de acesso; relato de dor de dente e na gengiva nos últimos 6 meses.

A seleção das variáveis explicativas para a construção do modelo multivariado foi feita segundo critérios de plausabilidade biológica e estatísticos, dentre aquelas com valores de intervalo de confiança significativos.

\section{Resultados}

Durante o processo de calibração, os resultados da concordância interexaminadores obtido pelo coeficiente kappa foi $0,760(0,713-0,806)$ e para a concordância intraexaminadores foi $0,935(0,871-0,998)$, uma substancial concordância ${ }^{17}$. Os indicadores apresentados foram considerados adequados para levantamento dessa natureza ${ }^{17,18}$.
Em 35 dos 780 domicílios sorteados, que poderiam conter adultos no grupo etário de 35 a 44 anos de idade, não foi possível contatar nenhum morador. Nos 745 domicílios onde foram estabelecidos contato com o morador, 237 apresentavam adultos no grupo etário de interesse e identificaram-se 336 indivíduos; destes, $12 \%$ (40), se recusaram a participar da pesquisa e $7 \%$ (23) não foram encontrados ou não estavam disponíveis para a realização da pesquisa, mesmo após a primeira visita e o retorno da equipe de examinadores em mais duas visitas com agendamento prévio. Assim, a taxa de não resposta dos adultos identificados foi de $19 \%$, esse percentual ficou abaixo do valor estimado para o cálculo da amostra.

Foram examinadas e entrevistadas 273 pessoas no grupo etário de 35 a 44 anos de idade, em 237 domicílios, ou seja, em 30,4\% dos domicílios sorteados. Após as entrevistas e os exames bucais, dez indivíduos foram excluídos por serem desdentados totais.

A amostra final para esta pesquisa foi, portanto, de 263 adultos, sendo 187 mulheres $(72 \%)$ e 76 homens (28\%). Em relação à cor da pele, $58,2 \%$ se declararam brancos e $41,8 \%$ se declararam negros ou pardos, quanto ao hábito de fumar $22,1 \%$ dos adultos declararam ser fumantes.

Apenas 10,6\% da amostra não apresentaram qualquer agravo periodontal em todos os sextantes examinados (Tabela 1); o sangramento foi a pior condição encontrada nos sextantes em 9,1\% dos indivíduos; pouco mais da metade dos indivíduos $(53,3 \%)$ apresentou o cálculo dentário em ao menos um sextante; bolsa periodontal rasa (3,5-5,5 $\mathrm{mm})$ esteve presente em quase um quarto da amostra $(22,4 \%)$ e apenas $4,2 \%$ apresentavam bolsa periodontal profunda (mais de $5,5 \mathrm{~mm}$ ).

Para o desfecho sangramento e cálculo foram analisados os dados de 193 adultos (comparando indivíduos com condições periodontais reversíveis em relação aos sadios), excluiu-se 70 indivíduos que apresentaram bolsa periodontal. Esse procedimento teve, como finalidade, evitar um viés nos resultados, pois a presença de bolsa 
Tabela 1. Distribuição de adultos (35 a 44 anos) residentes no município de Guarulhos (SP), 2006, segundo condições periodontais (maior grau do índice CPI)

Table 1. Distribution of adults (35 to 44 years old) living in the city of Guarulhos (SP), 2006, according to periodontal status (higher CPI score)

\begin{tabular}{lccc}
\hline Maior grau periodontal & $\mathrm{n}$ & $\%$ & IC 95\% \\
\hline Hígido & 28 & 10,6 & $7,3-14,8$ \\
Sangramento & 24 & 9,1 & $6,1-13,1$ \\
Cálculo dentário & 141 & 53,6 & $47,6-59,9$ \\
Bolsa periodontal rasa (4-5 mm) & 59 & 22,4 & $17,7-27,8$ \\
Bolsa periodontal profunda (>6 mm) & 11 & 4,2 & $2,2-7,2$ \\
Total & 263 & 100,0 & \\
\hline
\end{tabular}

CPI: Community Periodontal Index

periodontal é considerada uma condição irreversível da doença.

Para a análise do desfecho bolsa periodontal foram analisados os dados de 263 adultos (comparando-se 70 indivíduos com condições periodontais irreversíveis em relação às pessoas que apresentaram condições periodontais reversíveis e sadios), ambos os desfechos foram incluídos nos modelos bivariado e multivariado.

A análise estatística identificou que dentre as características demográficas, socioeconômicas e comportamentais individuais (Tabela 2), em relação à prevalência das condições periodontais para sangramento e cálculo, houve significância para os fumantes (RP=1,13; IC 95\% 1,02-1,26), pessoas com até oito anos de estudo $(\mathrm{RP}=1,17$; IC 95\% 1,04-1,33), que não frequentavam cirurgião-dentista há mais de dois anos ( $R P=1,19$; IC 95\% 1,06-1,35), e que relataram dor de dentes e gengiva nos últimos seis meses (RP=1,12; IC 95\% 1,01-1,24).

Para a prevalência de bolsa periodontal (rasa ou profunda) verificou-se associação para adultos fumantes (RP=1,78; IC 95\% 1,08-2,94), residentes em domicílios com maior aglomeração ( $\mathrm{RP}=2,35$; IC 95\% 1,23$4,50)$, e há mais de dois anos sem frequentar o cirurgião-dentista $(\mathrm{RP}=2,02$; IC $95 \%$ $1,10-3,75)$.

O tipo de serviço utilizado para a resolução de necessidades odontológicas (público ou privado) em relação à faixa etária de adultos mais jovens (35 a 39) ou mais velhos (40 a 44 anos) não demonstrou associação estatística com nenhum das condições pesquisadas.

O modelo de análise multivariada ajustado para amostras complexas e com ponderação populacional demonstrou que houve associação a um nível de significância de $95 \%$ do intervalo de confiança da Razão de Prevalência (Tabela 3). Para o desfecho de prevalência das condições de sangramento gengival e cálculo dentário houve associação significativa para homens ( $\mathrm{RP}=1,12$; IC 95\% 1,00-1,26), que relataram o hábito de fumar ( $\mathrm{RP}=1,11$; IC $95 \%$ 1,02$1,21)$, apresentavam até oito anos de estudo (RP 1,14; IC 95\% 1,02-1,28), não frequentavam o cirurgião-dentista há mais de dois anos (RP=1,19; IC 95\% 1,05-1,35).

Para o desfecho de bolsa periodontal rasa ou profunda houve associação para os adultos que relataram o hábito de fumar $(\mathrm{RP}=1,71$; IC 95\% 1,07-2,73), e cuja a última visita ao cirurgião-dentista foi superior a dois anos (RP=1,97; IC 95\% 1,06-3,69).

\section{Discussão}

Tendo em vista que a maioria dos estudos na área de saúde bucal são relativos à cárie dentária em escolares, a contribuição desta pesquisa consiste em analisar a condição periodontal baseada em um inquérito epidemiológico realizado em adultos em seus domicílios, com inferência populacional. Além da coleta das condições clínicas adicionou-se, em nível individual, um inquérito com 
Tabela 2. Associação de condições periodontais com variáveis demográficas, socioeconômicas e comportamentais em adultos (35 a 44 anos) residentes no município de Guarulhos (SP), 2006

Table 2. Association of periodontal status with demographic, socioeconomic and behavioral characteristics in adults (35 to 44 years old) in the city of Guarulhos (SP), 2006

\begin{tabular}{|c|c|c|c|c|c|c|c|c|c|c|}
\hline \multirow[t]{2}{*}{ Variáveis } & \multicolumn{6}{|c|}{$\begin{array}{l}\text { Sangramento genvival e } \\
\text { cálculo dentário ( } n=193)\end{array}$} & \multicolumn{4}{|c|}{ Bolsa periodontal $(n=263)$} \\
\hline & $\mathrm{n}$ & $\operatorname{sim}$ & não & $\mathrm{RP}$ & IC 95\% & $\mathrm{n}$ & $\operatorname{sim}$ & não & $\mathrm{RP}$ & IC 95\% \\
\hline \multicolumn{11}{|l|}{ Grupo etário } \\
\hline 40 a 44 anos & 193 & 75 & 15 & 0,967 & $0,86-1,07$ & 263 & 34 & 90 & 1,127 & $0,69-1,82$ \\
\hline$\%$ & & 38,9 & 7,8 & & & & 12,9 & 34,2 & & \\
\hline 35 a 39 anos & & 90 & 13 & & & & 36 & 103 & & \\
\hline$\%$ & & 46,6 & 6,7 & & & & 13,7 & 39,2 & & \\
\hline \multicolumn{11}{|l|}{ Sexo } \\
\hline Masculino & 193 & 47 & 4 & 1,088 & $0,96-1,22$ & 263 & 25 & 51 & 1,204 & $0,68-2,13$ \\
\hline$\%$ & & 24,4 & 2,1 & & & & 9,5 & 19,4 & & \\
\hline Feminino & & 118 & 24 & & & & 45 & 142 & & \\
\hline$\%$ & & 61,1 & 12,4 & & & & 17,1 & 54,0 & & \\
\hline \multicolumn{11}{|l|}{ Fumo } \\
\hline Fumante & 193 & 36 & 2 & 1,136 & $1,02-1,26$ & 263 & 20 & 38 & 1,781 & $1,08-2,94$ \\
\hline$\%$ & & 18,7 & 1,0 & & & & 7,6 & 14,4 & & \\
\hline Não fumante & & 129 & 26 & & & & 50 & 155 & & \\
\hline$\%$ & & 66,8 & 13,5 & & & & 19,0 & 58,9 & & \\
\hline \multicolumn{11}{|l|}{ Anos de Estudo } \\
\hline Até 8 anos & 193 & 98 & 10 & 1,178 & $1,04-1,33$ & 263 & 45 & 108 & 1,367 & $0,89-2,09$ \\
\hline$\%$ & & 50,8 & 5,2 & & & & 17,1 & 41,1 & & \\
\hline Mais de 8 anos & & 67 & 18 & & & & 25 & 85 & & \\
\hline$\%$ & & 34,7 & 9,3 & & & & 9,5 & 32,3 & & \\
\hline \multicolumn{11}{|l|}{ Aglomeração domiciliar } \\
\hline 1 ou mais pes/cômodo & 193 & 105 & 15 & 1,066 & $0,95-1,18$ & 263 & 57 & 120 & 2,354 & $1,23-4,50$ \\
\hline$\%$ & & 54,4 & 7,8 & & & & 21,7 & 45,6 & & \\
\hline Menos de 1 pes/cômodo & & 60 & 13 & & & & 13 & 73 & & \\
\hline$\%$ & & 31,1 & 6,7 & & & & 4,9 & 27,8 & & \\
\hline \multicolumn{11}{|l|}{ Dor nos últimos 6 meses } \\
\hline Relato de dor & 193 & 67 & 6 & 1,121 & $1,01-1,24$ & 263 & 29 & 73 & 1,379 & $0,72-2,65$ \\
\hline$\%$ & & 34,7 & 3,1 & & & & 11,0 & 27,8 & & \\
\hline Não relatou dor & & 98 & 22 & & & & 41 & 120 & & \\
\hline$\%$ & & 50,8 & 11,4 & & & & 15,6 & 45,6 & & \\
\hline \multicolumn{11}{|l|}{ Consulta ao dentista } \\
\hline 2 anos e mais & 193 & 88 & 8 & 1,199 & $1,06-1,35$ & 263 & 46 & 96 & 2,029 & $1,10-3,75$ \\
\hline$\%$ & & 45,6 & 4,1 & & & & 17,5 & 36,5 & & \\
\hline Menos de 2 anos & & 77 & 20 & & & & 24 & 97 & & \\
\hline$\%$ & & 39,9 & 10,4 & & & & 9,1 & 36,9 & & \\
\hline
\end{tabular}

RP: Razão de prevalência ajustado pelo modelo de amostragem por conglomerado e ponderação pelo peso populacional

$R P$ : prevalence ratio adjusted by cluster sampling model with weight population 
Tabela 3. Análise multivariada dos fatores associados à prevalência de condições periodontais em adultos residentes no município de Guarulhos (SP), 2006

Table 3. Multivariate analysis of factors associating with periodontal status in adults (35 to 44 years old) in the city of Guarulhos (SP), 2006

\begin{tabular}{lcccccc}
\hline \multirow{2}{*}{ Variáveis } & \multicolumn{3}{c}{ Sangramento genvival e cálculo dentário } & \multicolumn{3}{c}{ Bolsa periodontal } \\
\cline { 2 - 6 } & $\mathrm{RP}$ & $\mathrm{IC} 95 \%$ & $\mathrm{p}$ & $\mathrm{RP}$ & $\mathrm{IC} 95 \%$ & $\mathrm{p}$ \\
\hline Masculino & 1,127 & $1,00-1,26$ & 0,045 & - & - & - \\
Fumante & 1,115 & $1,02-1,21$ & 0,017 & 1,717 & $1,07-2,73$ & 0,023 \\
Até 8 Anos de Estudo & 1,148 & $1,02-1,28$ & 0,018 & - & - & - \\
Mais de 2 anos sem acesso ao CD & 1,194 & $1,05-1,35$ & 0,007 & 1,979 & $1,06-3,69$ & 0,033 \\
\hline
\end{tabular}

RP: Razão de prevalência ajustado pelo modelo de amostragem por conglomerado e ponderação pelo peso populacional CD: cirurgião-dentista

RP: prevalence ratio adjusted by Cluster Sampling model with weight population

$C D$ : dentist surgeon

variáveis demográficas, socioeconômicas e comportamentais.

Procurou-se, neste estudo, contemplar todas as etapas que pudessem validar a inferência estatística para base populacional. No resultado da análise dos dados da amostra por conglomerado, observou-se que o efeito do desenho foi de 1,309 para o desfecho sangramento gengival e cálculo dental; para o desfecho presença de bolsa periodontal foi de 1,918. Esses valores ficaram dentro dos limites preestabelecidos na fórmula para o cálculo da amostra (efeito do desenho=2,0). Korn e Grauband ${ }^{19}$ recomendam que o efeito do desenho tenha um valor de referência não superior a 1,5 , pois valores superiores a esses acabam tendo impacto sobre a precisão das estimativas se não forem levados em consideração o efeito do conglomerado, o que pode gerar alguma homogeneidade intraclasse e aumentar o erro padrão das estimativas. Sousa e Silva ${ }^{14}$ recomendam que o processo de análise dos resultados leve em consideração os modelos complexos de sorteio, e a utilização de programas estatísticos que possuam módulos específicos. Nesta pesquisa levou-se em consideração o efeito do conglomerado para o cálculo do tamanho da amostra e utilizaram-se programas específicos para a correção desse efeito com a geração de estimativas mais precisas.

Queiroz et al. ${ }^{20}$ criticam o resultado apresentado em levantamentos epidemiológicos de base populacional, que utilizam modelos de amostragem complexa na pesquisa, mas não levam em consideração na análise o delineamento do sorteio da amostra, a unidade primária de amostragem, como também não fazem ponderação populacional, o que compromete a inferência estatística populacional.

Em inquéritos domiciliares que apresentam conglomerados de tamanhos desiguais devido às etapas de sorteio para minimizar o efeito do desenho, utilizou-se o modelo de linearização de Taylor para análise dos resultados ${ }^{16}$. Foi ainda utilizado o peso de ponderação (weight) ${ }^{21}$ para ajustar a diferença de probabilidade de sorteios das unidades de amostragem.

Uma das limitações do instrumento empregado para o diagnóstico da condição periodontal é a dificuldade de definição do agravo periodontal expressa pela própria complexidade do agravo, visto que em um mesmo indivíduo regiões periodontais distintas apresentam condições com diferentes severidades, que vão desde um sextante hígido até outro com bolsa profunda. Para tanto, a descrição do agravo pode se dar por sextantes comprometidos ou pela pior condição apresentada pelo indivíduo, o que super estima o agravo, pois não se consideram os outros sextantes com menores gradientes de severidade.

A mensuração da condição periodontal pelo índice CPI é limitada, pois oferece uma visão parcial da doença e não leva em conta a evolução histórica do agravo ${ }^{22}$; 
subestima o agravo de menor severidade, pois, quando são identificados agravos como cálculo e bolsa, o sangramento gengival não é considerado ${ }^{23}$.

Se, por um lado, ocorreu uma grande prevalência das condições de sangramento e cálculo nesses indivíduos (62,7\%), foi observado um reduzido número de indivíduos que apresentasse, em pelo menos um dos sextantes examinados, a condição com bolsa periodontal profunda ou severa $(4,2 \%)$. Essa observação está em concordância com os achados em outros estudos nacionais e internacionais ${ }^{3,22,23}$. A maior discrepância observada dos adultos de Guarulhos em relação aos da região Sudeste diz respeito ao sextante hígido. Nesse estudo apenas 10,6\% não apresentaram doença periodontal, ao passo que na região Sudeste esse percentual foi de $23,0 \%^{4}$.

As altas prevalências de sangramento e cálculo expõem os adultos ao risco de desenvolvimento de doença periodontal que, juntamente com cárie dentária, são as causas mais comuns da perda precoce dos dentes. $\mathrm{O}$ edentulismo tem como consequência direta a perda da função mastigatória, o comprometimento da estética e de relacionamentos interpessoais.

Segundo Ferreira et al. ${ }^{24}$, a percepção das pessoas de que é natural a perda de todos os dentes com o envelhecimento pode indicar uma negligência das práticas de higiene bucal, estabelecendo um ciclo que leva progressivamente à perda dos dentes.

Análise realizada por Frazão et al. ${ }^{25} \mathrm{em}$ adultos no Estado de São Paulo em 1998, sobre perda precoce de dentes, identificou que os homens trabalhadores de escolas rurais, mais velhos, negros e que residiam em municípios de pequeno porte sem água fluoretada tinham associação estatisticamente significante para a perda dentária de mais de 12 dentes. Houve correlação em nível contextual com municípios que apresentaram alta taxa de analfabetismo, baixo IDH.

A perda dentária também esteve relacionada à baixa escolaridade, em mulheres mais velhas, residentes de área rural, com mais de cinco pessoas no domicílio e que procuraram acesso aos serviços de rede pública de saúde motivados por dor de dente $^{6}$.

Neste estudo, a opção de análise pelos modelos multivariados se justificou pela possibilidade de identificar a interação entre as variáveis para a análise dos desfechos e suas respectivas inter-relações de ajuste. A variável sexo não apresentou significância estatística no modelo bivariado mas, quando associado no modelo mulitvariado, ganhou significância, em concordância com estudo em adultos brasileiros, que identificou maior prevalência de doença periodontal entre homens ${ }^{26}$.

Nesta pesquisa não se observou diferença estatisticamente significante em relação à idade e à cor de pele nos adultos examinados, contrariando os achados de outros estudos. Negros e pardos apresentaram maior prevalência e severidade da doença periodontal ${ }^{26}$. A doença periodontal avançada esteve associada à idade em afrodescendente, pertencente à camada social com baixo poder econômico, diabético, fumante e com hábito de higiene oral precário ${ }^{27,28}$.

A procura pelos serviços de saúde bucal em adultos pode ser motivada pela dor ${ }^{4}$, por isso, essas variáveis foram consideradas para a construção dos modelos.

A dor de dente apresentou associação no modelo bivariado e perdeu significado no modelo multivarido. Borges et al. ${ }^{29}$ observaram em estudo com adolescentes que a dor de dentes e gengiva foi associada positivamente à presença de cálculo dentário aferido pelo índice CPI; essa condição se manteve mesmo após ajuste por cárie dentária, indicando que a dor nas gengivas está associada à condição de presença de cálculo dentário, independente de cárie.

Piores condições socioeconômicas estão associadas ao desfecho de doença periodontal, identificadas em estudos nacionais $^{30}$ e internacionais ${ }^{31,32}$. Neste estudo, a baixa escolaridade em adultos caracteriza a privação social desse grupo, e essa variável manteve associação no modelo 
multivariado para o desfecho sangramento gengival e cálculo dentário.

Também a presença da doença periodontal é maior em grupos específicos, como diabéticos ${ }^{33}$ e fumantes ${ }^{30,31,34}$.

Macedo $^{30}$, em estudo transversal em indivíduos de 20 a 60 anos de idade de uma área rural de Feira de Santana, Bahia, pela análise de regressão logística multivariada, identificou correlação entre prevalência de agravo periodontal e homens acima de 30 anos, residentes em domicílio com mais de uma pessoa por cômodo, fumantes ou ex-fumantes e com mais de quatro dentes ausentes.

Drumont-Santana et al. ${ }^{33}$, ao avaliarem agravos periodontais (sangramento e bolsa periodontal) e qualidade de vida (OHIP) em 159 diabéticos na cidade de Itaúna, Minas Gerais, descreveram que houve associação positiva, com impacto significativo na qualidade de vida dos indivíduos pesquisados.

Neste estudo, o hábito de fumar apresentou significância estatística multivariada com a prevalência de agravos periodontais e presença de bolsa periodontal. Estes resultados também foram identificados em adultos suecos por Nordery et al. ${ }^{31}$, que associaram a severidade do agravo periodontal a fatores socioeconômicos e fumo através de regressão logística multinível. A associação entre tabagismo e doença periodontal também foi encontrada entre jovens israelenses que se apresentaram para o serviço militar compulsório. $\mathrm{O} \mathrm{CPI}=0$ foi encontrado em $16 \%$ dos jovens, $\mathrm{CPI}=1$ e 2 em $78 \%$ e CPI=3 e 4 em $6 \%$. A condição periodontal foi melhor entre as mulheres não-fumantes ${ }^{34}$.

Neste estudo, o tempo de visita ao cirurgião-dentista superior a dois anos associou-se aos desfechos de doença periodontal reversível e irreversível. Estes resultados também foram encontrados em adultos na Alemanha por Krustrup e Erik-Petersen ${ }^{32}$, onde o agravo periodontal teve associação significativa com variáveis socioeconômicas e o tempo de visita ao cirurgião-dentista.

A baixa procura pelos serviços de saúde bucal pode estar associada à percepção das condições bucais, pois para algumas pessoas os problemas periodontais não são considerados doenças. Moreira et al. ${ }^{35}$ observaram que mesmo os idosos que apresentam uma condição clínica bucal desfavorável tiveram uma percepção positiva em relação a sua condição de saúde bucal. Segundo os autores, esse fato cria uma falácia subjetiva pelo fato de que os idosos, apesar de terem perdido os dentes, não se consideram como mutilados dentários, reforçado o pensamento de que a perda dentária é um processo natural do envelhecimento ${ }^{24}$.

Outro fator limitador da baixa demanda dos serviços de saúde pelos adultos é que, por algumas décadas, o modelo hegemônico de saúde bucal no serviço público estava restrito ao atendimento de crianças matriculadas em escolas públicas e, nos poucos serviços de saúde que ofereciam assistência, predominavam o atendimento de urgência com ações de exodontias, levando precocemente muitos adultos ao edentulismo.

Mesmo após a implantação do Sistema Único de Saúde, a municipalização dos serviços, a universalização do acesso a todos os segmentos etários e a extensão da cobertura por meio do Programa de Saúde da Família, os serviços de saúde bucal não conseguem ser resolutivos ${ }^{36}$ em atender a demanda reprimida por décadas de descaso social. O uso dos serviços públicos odontológicos pela população adulta parece ainda ser restrito. A maior utilização regular desse ocorre entre os mais escolarizados ${ }^{37,38}$.

Observou-se que os moradores de Guarulhos que não completaram o ensino fundamental apresentaram maior prevalência de sangramento e cálculo dental, mantendo-se como variável explicativa no modelo multivariado conjuntamente com as variáveis sexo, hábito de fumar e tempo de consulta ao dentista. A baixa escolaridade também foi identificada em adultos no Brasil ${ }^{26,39}$ e está relacionada com a perda precoce de dentes e o edentulismo ${ }^{6,11}$. Há uma relação consistente entre a baixa escolaridade e condições socioeconômicas desfavoráveis, o que leva essas pessoas a terem menor acesso a produtos de higiene 
bucal $^{40,41}$. Essa conjugação de fatores amplia o risco de problemas periodontais.

Os dados deste estudo indicaram uma maior prevalência das condições sangramento gengival e cálculo dentário, que tem caráter reversível, e que podem ser sanados por procedimentos da atenção básica em saúde bucal, nas Unidades Básicas de
Saúde. Assim sendo, o acesso dessa população aos serviços de saúde do município deveriam ser ampliados ou facilitados e, buscar referências para os serviços de especialidades odontológicas ampliaria a resolutividade para esse agravo, o que poderia evitar a perda precoce dos dentes e a mutilação dentária.

\section{Referências}

1. Gugushe TS. The influence of sócio-economic variables on the prevalence of periodontal disease in South Africa. SADJ. 1998;53(2):41-6.

2. Papapanou PN. Periodontal diseases: epidemiology. Ann Periodontal. 1996;1:1-36.

3. Phipps KR, Stephens VJ. Relative contribution of caries and periodontal disease in adult tooth loss for an HMO dental population. J Public Health Dent. 1995;55:250-2.

4. Brasil. Ministério da Saúde. Secretaria de Atenção à Saúde. Departamento de Atenção Básica. Projeto SB Brasil 2003: condições de saúde bucal da população brasileira 2002-2003. Resultados principais. Brasília: Ministério da Saúde; 2004.

5. SEADE - Fundação Sistema Estadual de Análise de Dados. Perfil Municipal. [internet]. [citado 15 abr 2008]. Disponível em: <http://www.seade.gov.br/produtos/perfil/perfil.php>.

6. Silva DD, Rihs LB, Sousa MLR. Fatores associados à presença de dentes em adultos de São Paulo, Brasil. Cad Saúde Pública. 2009;25(11):2407-18.

7. Barbato PR, Nagano HCM, Zanchet FN, Boing AF, Peres MA. Perdas dentárias e fatores sociais, demográficos e de serviços associados em adultos brasileiros: uma análise dos dados do Estudo Epidemiológico Nacional (Projeto SB Brasil 2002-2003). Cad Saúde Pública. 2007;23(8):1803-14.

8. Matos DL, Lima-Costa MF. Auto-avaliação da saúde bucal entre adultos e idosos residentes na Região Sudeste: resultados do Projeto SB-Brasil, 2003. Cad Saúde Pública. 2006;22(8):1699-1707.

9. Vargas AMD, Paixão HH. Perda dentária e seu significado na qualidade de vida de adultos usuários de serviço público de saúde bucal do Centro de Saúde Boa Vista, em Belo Horizonte. Ciênc \& Saúde Coletiva. 2005;10(4):1015-24.

10. Gomes AS, Abegg C. O impacto odontológico no desempenho diário dos trabalhadores do Departamento Municipal de Limpeza Urbana de Porto Alegre, Rio Grande do Sul, Brasil. Cad Saúde Pública. 2007;23(7):1707-14.

11. Frazão P, Antunes JLF, Narvai PC. Perda dentária precoce em adultos de 35 a 44 anos de idade: Estado de São Paulo, Brasil, 1998. Rev Bras Epidemiol. 2003;6(1):49-57.
12. Lwanga SK, Lemeshow S. Sample size determination in health studies. A pratical manual. Geneva: World Health Organization; 1991.

13. Silva NN. Amostragem probabilística. São Paulo: Edusp; 1998.

14. Sousa MH, Silva NN. Estimativas obtidas de um levantamento complexo. Rev Saúde Pública. 2003;37(5):622-70.

15. World Health Organization. Oral health surveys: basic methods. $4^{\text {th }}$. ed. Geneva: 1997.

16. Alves MCGP, Silva NN. Métodos de estimação de variância em amostras provenientes de inquéritos domiciliares. Rev Saúde Pública. 2007;51(6):938-46.

17. Landis JR, Kock GG. The measurement of observer agreement for categorical date. Biometrics. 1977;33:159-74.

18. Frias AC, Antunes JLF, Narvai PC. Precisão e validade de levantamentos epidemiológicos em saúde bucal: cárie dentária na Cidade de São Paulo, 2002. Rev Bras Epidemiol. 2004;7(2):144-54.

19. Korn EL, Graubard BI. Epidemiologic studies utilizing surveys: accounting for the sampling design. Am J Public Health. 1991;81:1166-73.

20. Queiroz RCS, Portela MC, Vasconcelos MTL. Pesquisa sobre condições de saúde bucal da população brasileira (SB Brasil 2003): Seus dados não produzem estimativas populacionais, mas há possibilidades de correção. Cad Saúde Pública. 2009;25(1):47-58.

21. Pedroso GC, Puccini RF, Silva EMK, Silva NN, Alves MCGP. Prevalência de aleitamento materno e introdução precoce de suplementos em área urbana do sudeste do Brasil, EMBU, SP. Rev Bras Saúde Materno Infantil. 2004;4(1):45-58.

22. Tomita NE, Chinellato LEM, Pernambuco RA, Lauris JR, Franco LJ. Condições periodontais e diabetes mellitus na população nipo-brasileira. Rev Saúde Pública. 2002;36(5):607-13.

23. Gesser HC, Peres MA, Marcenes W. Condições gengivais e periodontais associadas a fatores socioeconômicos. Rev Saúde Pública. 2001;35(3):289-93. 
24. Ferreira RC, Magalhaes CS, Rocha ES, Schwambach CW, Moreira AN. Saúde bucal de idosos residentes em instituições de longa permanência de Belo Horizonte, Minas Gerais Brasil. Cad Saúde Pública. 2009;25(11):2375-85.

25. Frazão P, Antunes JLF, Narvai PC. Perda precoce em adultos de 35 a 44 anos de idade. Estado de São Paulo, Brasil, 1998. Rev Bras Epidemiol. 2003;6(1):49-57.

26. Peres MA, Antunes JLF, Boing AF, Peres KG, Bastos JLD. Skin colour is associated with periodontal disease in Brazilian adults: a population-base oral health survey. J Clin Periodontol. 2007;34:196-201.

27. Genco RJ. Current view of risk factors for periodontal diseases. J Periodontol. 1996;67:1041-9.

28. Papapanou PN, Lindhe J. Epidemiologia da doença periodontal. In: Lindhe J. Tratado de periodontia clinica e implantodontia oral. $3^{\text {a }}$ ed. Rio de Janeiro: Guanabara Koogan; 1999. p. 42-62.

29. Borges CM, Cascaes AM, Fischer TK, Boing AF, Peres MA, Peres KG. Dor nos dentes e gengivas e fatores associados em adolescentes brasileiros: análise de inquérito nacional de saúde bucal SBBrasil 2002-2003. Cad Saúde Pública. 2008;24(8):1825-34.

30. Macedo TCN, Costa MCN, Gomes- Filho IS, Viana MIP, Santos CT. Factors related to periodontal disease in a rural population. Braz Oral Res. 2006;20(3):257-62.

31. Nordery O, Hugoson A, Grusovin G. Risk of severe periodontal disease in a Swedish adult population. A longitudinal study. J Clin Periodonal. 1999;26(9):608-15.

32. Krustrup U, Erik Petersen P. Periodontal conditions in 35-44 and 65-74-year-old adults in Denmark. Acta Odontol Scand. 2006;64(2):65-73.

33. Drumont-Santana T, Costa FO, Zenóbio EG, Soares RV, Santana TD. Impacto da doença periodontal na qualidade de vida de indivíduos diabéticos dentados. Cad de Saúde Pública. 2007;23(3):637-44.

34. Vered Y, Livny A, Zini A, Sgan-Cohen HD. Periodontal health status and smoking among young adults. J Clin Periodontol. 2008;35(9):768-72.

35. Moreira RS, Nico LS, Souza MLR. Fatores associados à necessidade subjetiva de tratamento odontológico em idosos brasileiros. Cad Saúde Pública. 2009;25(12):2661-71.

36. Rocha RACP, Góes PSA. Comparação do acesso aos serviços de saúde bucal em áreas coberta e não cobertas pela Estratégia Saúde da Família em Campina Grande, Paraíba, Brasil. Cad Saúde Pública. 2008;24(12):28671-80.

37. Araújo CS, Lima RC, Peres MA, Barros AJD. Utilização de serviços odontológicos e fatores associados: um estudo de base populacional no Sul do Brasil. Cad Saúde Pública. 2009;25(5):1063-72.

38. Camargo MBJ, Dumith SC, Barros AJD. Uso regular de serviços odontológicos entre adultos: padrões de utilização e tipos de serviços. Cad Saúde Pública 2009, 25(9):1894-1906.

39. Segundo TK, Ferreira EF, Costa JE. A doença periodontal na comunidade negra do Arturo' s, Contagem, Minas Gerais, Brasil. Cad Saúde Pública. 2004;20(2):596-603.

40. Abegg C. Hábitos de higiene bucal dos adultos portoalegrenses. Rev Saúde Pública. 1977;31:586-93.

41. Daviglio RS, Aerts DRGC, Abegg C, Freddo SL, Monteiro L. Fatores associados a hábitos de saúde bucal e utilização de serviços odontológicos em adolescentes. Cad Saúde Pública. 2009;25(3):655-67.

Recebido em: 06/08/2009

Versão final apresentada em: 22/03/2011

Aprovado em: 05/06/2011 